\title{
Diaphragmatic hernia presenting in adult life
}

\author{
Peiris $\mathrm{SPM}^{1}$, Amaratunga $\mathrm{HA}^{2} *$, Seneviratne $\mathrm{RJK}^{3}$, Lamawanse MD \\ ${ }^{I}$ Department of Surgery, Faulty of Medicine, University of Peradeniya. \\ ${ }^{2}$ Department of Anatomy, Faulty of Medicine, University of Peradeniya. \\ ${ }^{3}$ Surgical Unit, Teaching Hospital Kandy. \\ *himanipeiris@yahoo.com
}

Diaphragmatic hernia (DH) usually presents in the newborn and rarely present as undetected cases in adult life. Defects in the diaphragm can cause abdominal organs to herniate into the thoracic cavity resulting in respiratory distress or strangulation of abdominal organs. Reported here are 3 cases of congenital DH presenting in adult life.

A 59-year-old male presenting with epigastric pain, bilious vomiting and constipation demonstrated an air-fluid level in the anterior mediastinum on chest radiograph. Surgery revealed an anterior Morgagni defect with a loop of terminal ileum herniated and strangulated into the pericardial sac. A 13-year-old girl presenting with abdominal pain and pyrexia for 2 days, on $\mathrm{CT}$ chest and abdomen revealed right lobe of liver and transverse colon in the right hemithorax with the pericardium pushed to the opposite side. Surgery revealed a large right sided central tendon defect. A strangulated gangrenous transverse colon was removed from within the thoracic cavity. A 73-year-old woman presented respiratory distress and was found to have left sided pleural effusion and bowel shadows in the chest cavity on radiography. CT revealed the stomach and several small intestinal loops herniating through a posterior defect in the left hemi-diaphragm. In these cases the body had adapted by maintaining total lung volume by compensatory hyperplasia of the contralateral lung. It was fascinating to find the liver moulded to the shape of the thoracic cavity in case 2 . It appeared that the herniation of bowel loops had occurred as an acute event giving rise to symptoms. Even though rare, the possibility of congenital DH presenting as acute abdomen or respiratory distress is important to remember. 